\title{
A EXPOSIÇÃO OCUPACIONAL A VIBRAÇÕES DE CORPO INTEIRO EM TRATOR AGRÍCOLA COM
} SUBSOLADOR

\author{
Ana Carolina Cazani ${ }^{(1)}$ (carolina.cazani@unesp.br), João Eduardo Guarnetti dos Santos ${ }^{(2)}$ \\ (joao.guarnetti@unesp.br) \\ (1) Universidade Estadual Paulista "Júlio de Mesquita Filho" - (UNESP); Departamento de Engenharia Mecânica \\ (2) Universidade Estadual Paulista "Júlio de Mesquita Filho" - (UNESP); Departamento de Engenharia Mecânica
}

RESUMO: As vibrações mecânicas transmitidas durante a operação de subsolagem do solo podem causar danos à saúde dos operadores. Assim, esse trabalho objetivou avaliar a vibração ocupacional atuante em um operador de trator agrícola com implemento subsolador acoplado enquanto executava a operação de subsolagem em uma área destinada ao plantio de eucalipto. Os procedimentos técnicos adotados foram os estabelecidos pela Norma de Higiene Ocupacional 09: Avaliação da exposição ocupacional a vibrações de corpo inteiro da Fundacentro. O instrumental consistiu em um analisador de vibração da Larson Davis modelo HVM 200 e um acelerômetro triaxial do tipo Seat Pad, que foi fixado no assento do trator agrícola com fita adesiva. Os ensaios foram realizados em duas áreas de terreno com variação na quantidade de resíduo florestal e umidade. Os ensaios demonstraram valores de aceleração resultante de exposição normalizada superiores ao limite de tolerância em ambos os cenários, indicando a necessidade de adoção imediata de medidas corretivas.

PALAVRAS-CHAVE: ERGONOMIA, SILVICULTURA, VIBRAÇÃO.

\section{OCCUPATIONAL EXPOSURE TO WHOLE-BODY VIBRATION IN TRACTOR WITH SUBSOILER}

ABSTRACT: Mechanical vibrations transmitted during subsoil operation may cause damage to the health of operators. Therefore, this work aimed to evaluate the occupational vibration acting on an agricultural tractor operator with attached subsoiler implement while performing the subsoiling operation in an area intended for eucalyptus planting. The technical procedures adopted were those established by Occupational Hygiene Standard 09: Assessment of Occupational Exposure to Fundacentro Full Body Vibrations. The instrument consisted of a Larson Davis model HVM 200 vibration analyzer and a Seat Pad triaxial accelerometer, which was attached to the farm tractor seat with tape. The tests were performed in two areas of terrain with varying amount of forest residue. The tests demonstrated acceleration values resulting from normalized exposure above the tolerance limit in both scenarios, indicating the need for immediate corrective action.

KEYWORDS: ERGONOMICS, SILVICULTURE, VIBRATION. 


\section{$\mathrm{XIX}$ CONEMI}

\section{INTRODUÇÃO}

A mecanização compreende todos os níveis de tecnologias agrícolas, não limitando-se aos equipamentos motorizados e sofisticados, mas também ferramentas manuais mais simples. A Organização das Nações Unidas para Alimentação e Agricultura (FAO, 2016) cita como algumas das vantagens da mecanização a diminuição do trabalho penoso, a redução da necessidade de mão-deobra, aumento da produtividade e pontualidade, melhoria na utilização de recursos e no acesso ao mercado. Paralelamente a essas alterações e melhorias, ocorreram naturalmente mudanças nas formas como o trabalho é realizado e da própria relação do trabalhador com este. Quando o equipamento é capaz de proporcionar conforto ao operador, é possível a ocorrência de aumento da produtividade, porém, quando ele não está adequado, ocorre o aumento do número de acidentes e doenças ocupacionais, principalmente por causa da exposição a problemas ergonômicos (SOUZA et al., 2004).

A Associação Brasileira de Ergonomia (2000) adota a seguinte definição para Ergonomia:

Ergonomia (ou Fatores Humanos) é a disciplina científica que trata da compreensão das interações entre os seres humanos e outros elementos de um sistema, e a profissão que aplica teorias, princípios, dados e métodos, a projetos que visam otimizar o bem estar humano e a performance global dos sistemas.

Através da aplicação das concepções dispostas pela ergonomia é possível aprimorar o desempenho de um sistema e a satisfação e bem-estar do ser humano. De acordo com lida e Buarque (2016), a Ergonomia além de estudar os variados fatores que podem atuar no funcionamento do sistema produtivo, busca a redução das consequências lesivas sobre o empregado auxiliando no desenvolvimento de melhorias de projeto, de condições e organização do trabalho, objetivando entre outros fatores a preservação da saúde e segurança do trabalhador.

Dentre os problemas ergonômicos que podem ser encontrados em atividades relacionadas ao agronegócio encontra-se a vibração. Para Rao (2008) vibração é um movimento que se repete após um intervalo de tempo. Segundo lida e Buarque (2016) é qualquer movimento oscilatório que o corpo ou parte dele executa em torno de um ponto. Para esses mesmos autores, algumas vibrações de natureza ocupacional são incômodas e indesejáveis, e como consequência podem afetar o desenvolvimento de algumas tarefas e causar lesões e doenças.

Usualmente as vibrações transmitidas ao corpo humano são classificadas de duas formas: vibrações de corpo inteiro e vibrações de mãos e braços. As vibrações de corpo inteiro (VCI) são transmitidas ao corpo como um todo, possuem baixa frequência e alta amplitude, situando-se na faixa de 1 a $80 \mathrm{~Hz}$ e são encontradas geralmente em atividades de transporte. Já as vibrações de 


\section{$\mathbf{X I X}$ \\ C.ONEMI}

mãos e braços (VMB) atingem certas extremidades do corpo, principalmente mãos e braços, apresentam uma faixa de frequência de 6,3 a $1250 \mathrm{~Hz}$ e ocorrem principalmente nas operações com ferramentas manuais (SALIBA, 2016). Em algumas pesquisas relacionadas à VCl foram encontradas evidências de que intensas vibrações de grande duração podem prejudicar a coluna vertebral e potencializar o risco de doenças lombares (VENDRAME, 2017). Já as VMB estão associadas com uma diversidade de distúrbios vasculares, neurológicos, articulares e muscoesqueléticos.

Assim, considerando a importância de se estudar os aspectos ergonômicos relacionados ao agente vibração atuantes no posto de trabalho de um operador, foi avaliado um trator com implemento do tipo subsolador em dois tipos de condições operacionais (variação da quantidade de resíduo de colheita). Com isso, foi possível caracterizar a vibração ocupacional atuante sob o trabalhador e sugerir melhorias para que se proporcione melhores condições de trabalho.

\section{MATERIAIS E MÉTODOS}

O procedimento experimental foi realizado em uma região destinada ao plantio de florestas com eucalipto e consistiu na avaliação da exposição ocupacional a vibração de corpo inteiro em um operador de trator agrícola (Figura 1) utilizado no preparo do solo. Os ensaios foram realizados em duas áreas de terreno com variação na quantidade de resíduo florestal e umidade do solo, caracterizadas nesse trabalho como Ensaio A (com maior quantidade de resíduo e umidade) e Ensaio B (com os dois parâmetros inferiores). Foi acoplado ao equipamento um implemento do tipo subsolador, com as seguintes características: implemento subsolador,marca Colossus Florestal e capacidade de carga de $600 \mathrm{~kg}$ para insumos, contendo haste parabólica e 8 discos de aiveca. Já o trator agrícola é da marca Valtra, modelo BH e potência de $180 \mathrm{cv}$. O total de horas acumuladas do equipamento é de 7.148 .

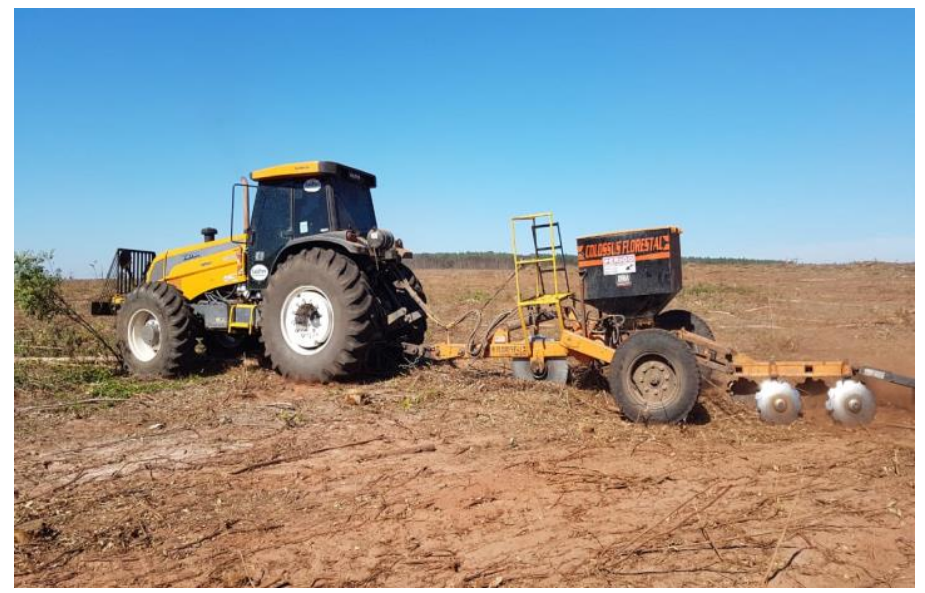

FIGURA 1. Trator com implemento subsolador utilizado no ensaio. Fonte: Autores (2019). 


\section{XIX \\ CENEMI}

Foram aplicados os seguintes parâmetros nos ensaios: subsolagem a $40 \mathrm{~cm}$ de profundidade e implemento com carga máxima de fertilizantes. Os terrenos são caracterizados como planos, isto é, declividade de 0 a 3\%. O mesmo trabalhador operou o equipamento em ambos os testes. Ele possuía massa de $100 \mathrm{~kg}$ e 1,78 m de altura. Os equipamentos de proteção individual foram fornecidos pelo empregador e consistiam em botina de segurança, protetor auricular, óculos de segurança, perneira e capuz de segurança.

Para a coleta dos dados da vibração atuante no operador, foi utilizado um analisador de vibração da marca Larson Davis. Ele atende os requisitos definidos pela norma ISO 8041 (2005) que determina os padrões a serem seguidos pelos instrumentos de medição projetados para medir valores de vibração, com o propósito de avaliar a resposta humana. Os procedimentos técnicos aplicados na avaliação de VCl foram os definidos pela Fundacentro através da Norma de Higiene Ocupacional 09: Avaliação da exposição ocupacional a vibrações de corpo inteiro (NHO 09). Essa normativa determina que as medições da vibração transmitida ao corpo devem ser feita de acordo com as três direções de um sistema de coordenadas ortogonais de forma simultânea, utilizando-se um acelerômetro do tipo triaxial.

Em conformidade com o determinado com a NHO 09 (2013), foi conectado ao analisador de vibração um acelerômetro triaxial ICP do tipo Seat Pad, modelo SEN027 (SN P236310), com a seguinte sensibilidade nominal: eixo $x=10,26 \mathrm{mV} / \mathrm{m} / \mathrm{s}^{2}$; eixo $\mathrm{y}=10,26 \mathrm{mV} / \mathrm{m} / \mathrm{s}^{2}$; eixo $z=10,33$ $\mathrm{mV} / \mathrm{m} / \mathrm{s}^{2}$ (Figura 2a). O Seat Pad foi fixado no assento do trator agrícola com fita adesiva do tipo de empacotamento, sem comprometer os resultados obtidos, respeitando a localização dos eixos, e permitindo que o operador execute suas atividades normalmente, conforme a Figura $2 \mathrm{~b}$ e Figura 2c. As avaliações foram realizadas em condições reais de operação durante 20 minutos, totalizando 1.200 registros em cada ensaio.
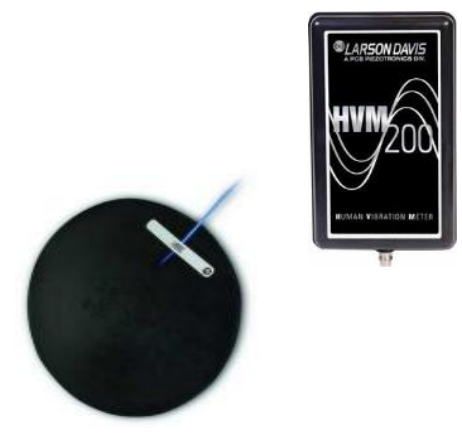

(a)

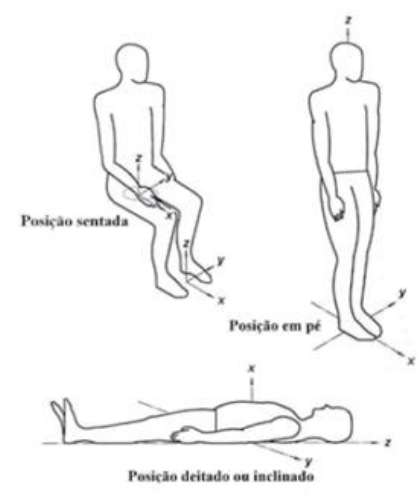

(b)

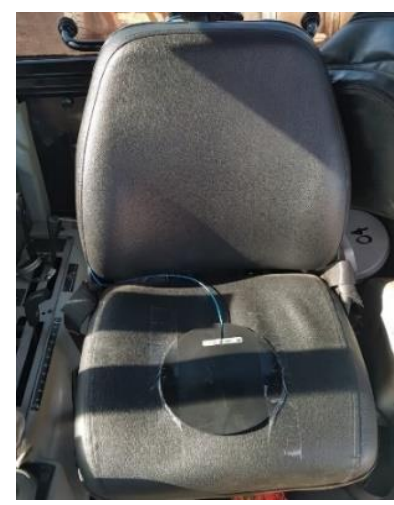

(c)

FIGURA 2. (a) Seat Pad e módulo de aquisição de dados - HVM 200. Fonte: Autores (2019).

(b) Orientação dos eixos conforme NHO 09 (2013).

(c) Acelerômetro fixado no assento de acordo com NHO 09 (2013). 


\section{$\mathbf{X I X}$ \\ C.ONEMI}

As avaliações dos dados técnicos foram baseadas na NHO 09 (2013). Ela fixa um sistema de coordenadas com origem na interface entre a fonte vibratória e o corpo humano. A aceleração média resultante ( $a m r)$ é calculada de acordo com a Equação 1 onde amjcorresponde a aceleração média de cada eixo e o $f_{j}$ é o fator de multiplicação em função do eixo considerado $(f=1,4$ para os eixos " $x$ " e " $y$ " e $f=1,0$ para o eixo " $z$ ").

$$
\mathrm{a} m r=\sqrt{\left(f_{x} \times a m_{x}\right)^{2}+\left(f_{y} \times a m_{y}\right)^{2}+\left(f_{z} \times a m_{z}\right)^{2}} \quad\left[\mathrm{~m} / \mathrm{s}^{2}\right]
$$

A aceleração resultante da exposição (are) é definida pela NHO 09 (2013) como sendo " $a$ aceleração média resultante representativa da exposição ocupacional diária" sendo mensurada através da Equação 2. O valor arep; corresponde a aceleração resultante da exposição parcial; ni é o número de repetições da componente de exposição "i" ao longo da jornada de trabalho; $T_{i}=$ Tempo de duração da componente de exposição “; $m$ = número de componentes de exposição que compõem a exposição diária; T= tempo da duração da jornada diária de trabalho.

$$
\text { are }=\sqrt{\frac{1}{\mathrm{~T}} \sum_{\mathrm{i}=1}^{\mathrm{m}} \mathrm{n}_{\mathrm{i}} \operatorname{arep}_{\mathrm{i}}^{2} \mathrm{~T}_{\mathrm{i}}} \quad\left[\mathrm{m} / \mathrm{s}^{2}\right]
$$

A aceleração resultante de exposição normalizada (aren) corresponde ao valor do are convertido para uma jornada diária padrão de 8 horas (NHO 09, 2013). Ela é calculada através da Equação 3. Em que $T$ = tempo de duração da jornada diária de trabalho expresso em horas ou minutos; $T_{0}=8$ horas ou 480 minutos.

$$
\text { aren }=\text { are } \times \sqrt{\frac{T}{T_{0}}} \quad\left[\mathrm{~m} / \mathrm{s}^{2}\right]
$$

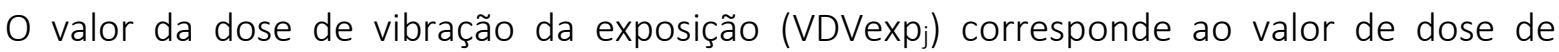
vibração representativo da exposição ocupacional diária em cada eixo de medição e pode ser obtido através da Equação 4, onde o VDVexpji refere-se a esse valor representativo em cada eixo e o $m$ refere-se ao número de componentes de exposição que compõem a exposição diária.

$$
V D V \exp _{j}=\left[\sum_{i=1}^{m}\left(V D V \exp _{j i}\right)^{4}\right]^{\frac{1}{4}} \quad\left[\mathrm{~m} / \mathrm{s}^{1,75}\right]
$$




\section{$\mathrm{XIX}$ \\ CENEMI}

Já o valor da dose de vibração resultante (VDVR) deve ser calculado por meio da Equação 5, onde VDVexpj é o valor da dose de vibração da exposição diária e segundo a NHO 09 (2013) considera a resultante dos três eixos de medição.

$$
V D V R=\left[\sum_{J}\left(V D V \exp _{j}\right)^{4}\right]^{\frac{1}{4}} \quad\left[\mathrm{~m} / \mathrm{s}^{1,75}\right]
$$

Com os valores encontrados para o aren e o VDVR, de acordo com a NHO 09 (2013), deve-se consultar a Tabela 1 que estabelecem os critérios de julgamento e tomadas de decisão. Assim, será possível determinar as medidas que devem ser consideradas para se controlar os níveis de exposição a vibração no operador.

TABELA 1. Critério de julgamento e tomada de decisão.

\begin{tabular}{|c|c|c|c|}
\hline $\begin{array}{c}\text { aren } \\
\left(\mathrm{m} / \mathrm{s}^{2}\right)\end{array}$ & $\begin{array}{c}\text { VDVR } \\
\left(\mathrm{m} / \mathrm{s}^{1,75}\right)\end{array}$ & $\begin{array}{l}\text { Consideração } \\
\text { técnica }\end{array}$ & Atuação recomendada \\
\hline 0 a 0,5 & 0 a 9,1 & Aceitável & $\begin{array}{l}\text { No mínimo manutenção da } \\
\text { condição existente. }\end{array}$ \\
\hline$>0,5 a<0,9$ & $>9,1 \mathrm{a}<16,4$ & $\begin{array}{l}\text { Acima do nível de } \\
\text { ação }\end{array}$ & $\begin{array}{l}\text { No mínimo adoção de medidas } \\
\text { preventivas. }\end{array}$ \\
\hline 0,9 a 1,1 & 16,4 a 21 & $\begin{array}{l}\text { Região de } \\
\text { incerteza }\end{array}$ & $\begin{array}{l}\text { Adoção de medidas preventivas e } \\
\text { corretivas visando à redução da } \\
\text { exposição diária. }\end{array}$ \\
\hline Acima de 1,1 & Acima de 21 & $\begin{array}{l}\text { Acima do limite } \\
\text { de exposição }\end{array}$ & $\begin{array}{l}\text { Adoção imediata de medidas } \\
\text { corretivas. }\end{array}$ \\
\hline
\end{tabular}

Fonte: NHO 09 (2013).

Com o objetivo de avaliar a dispersão e variabilidade dos dados foram construídos com gráficos boxplot por meio do aplicativo R versão 3.5.2 (R Development Core Team, 2018).

\section{RESULTADOS}

Os resultados dos ensaios estão apresentados a seguir, onde é possível notar na Figura 3, que faz referência a uma análise de dispersão dos dados, que o Ensaio A apresenta uma maior 


\section{$\mathbf{X I X}$ \\ CENEMI}

FENEMI

dispersão, comportamento que pode ser atribuído ao estado do terreno do ensaio A (bordadura do talhão com resíduo de colheita florestal). Outro aspecto que exerceu influência nessa maior dispersão foi a umidade do solo, sendo que para o Ensaio A o teor de umidade médio ( $W_{\text {médio }}$ foi

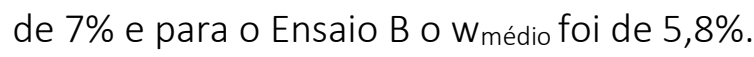

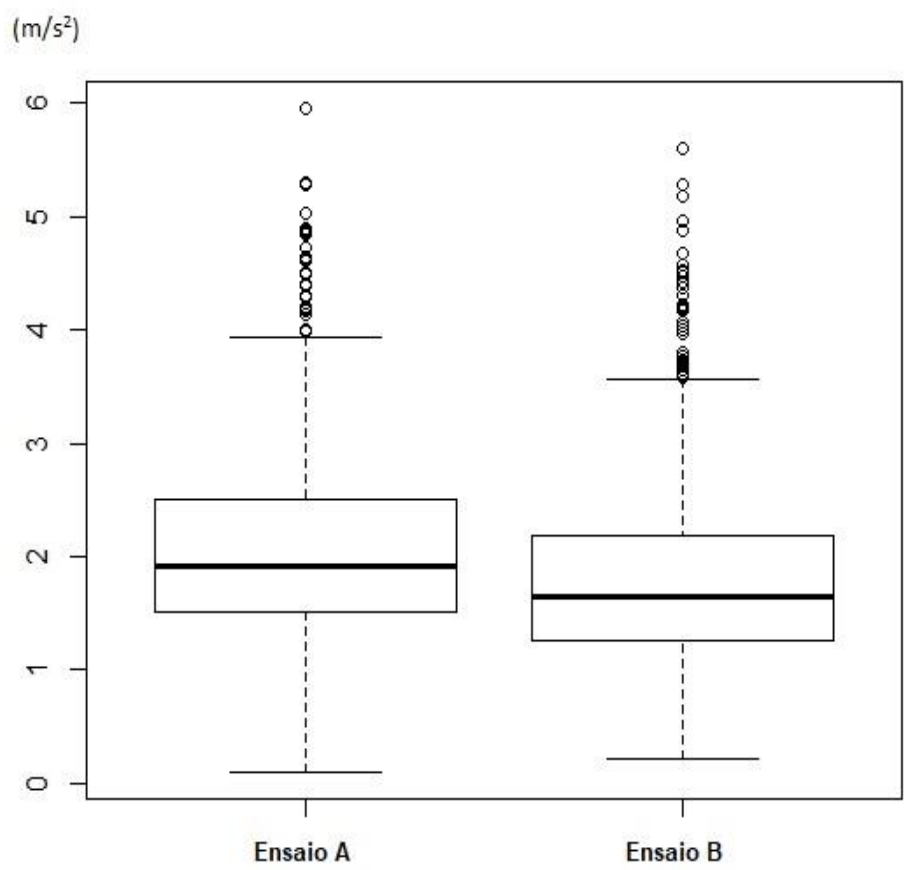

FIGURA 3. Comportamento da somatória ponderada dos três eixos no Ensaio A e no Ensaio B. Fonte: Autores (2019).

Avaliando-se a variação da somatória ponderada dos três eixos no decorrer do tempo para o Ensaio A e para o Ensaio B (Figura 4), constatou-se que os maiores valores de picos foram para a área com maior quantidade de resíduo da colheita de madeira, devido a maior dificuldade da realização da operação de subsolagem para esta condição operacional. Outro fator diretamente influenciado pela presença do resíduo da colheita de madeira foi observado na aceleração ponderada predominante do Eixo Y (Eixo X: 0,8807 m/s², Eixo Y: 1,1304 m/s², Eixo Z: 0,8613 m/s²).

No Ensaio B (área sem resíduo da colheita de madeira), a predominância da aceleração ponderada foi averiguada no Eixo $Y$, porém a diferença foi inferior (Eixo X: 0,7457 m/s², Eixo Y: $1,0126 \mathrm{~m} / \mathrm{s}^{2}$, Eixo Z: 0,8718 m/s $/ \mathrm{s}^{2}$ quando comparada ao Ensaio A. Também foi constatada, a presença de picos de alta amplitude no Ensaio $A$ em relação ao Ensaio $B$, o que pode ser explicado pela presença dos outliers da Figura 3. 


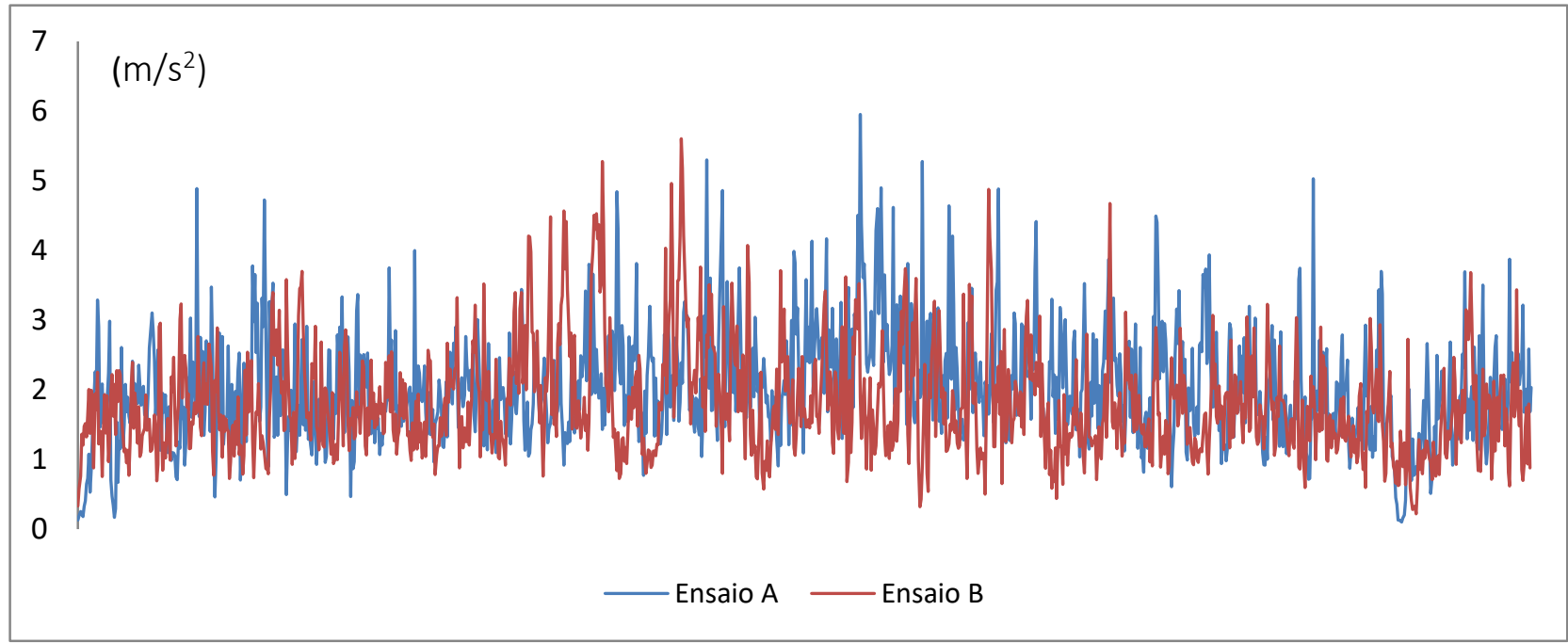

FIGURA 4. Comportamento da somatória ponderada dos três eixos no Ensaio A e no Ensaio B. Fonte: Autores (2019).

Os valores mensurados foram compilados na Tabela 2. Nota-se que mesmo com a variação na quantidade de resíduo florestal e umidade entre os terrenos dos ensaios, as diferenças não foram significativas tanto para o aren quanto para o VDVR. Comparando-se com a Tabela 1, que define o critério de julgamento e tomada de decisão, verifica-se que os valores referentes a dose de vibração (VDVR) são superiores ao limite de exposição nas duas condições (> $21 \mathrm{~m} / \mathrm{s}^{1,75}$ ). Já com relação aos valores da aceleração resultante de exposição normalizada, tanto o Ensaio A quanto o $\mathrm{B}$ também apresentaram valores maiores que o limite de tolerância $\left(1,1 \mathrm{~m} / \mathrm{s}^{2}\right)$. Assim, independente do critério adotado a atuação recomendada pela normativa é a imediata adoção de medidas corretivas.

TABELA 2. Dados compilados.

\begin{tabular}{l|c|c}
\hline & Aren $\left(\mathrm{m} / \mathrm{s}^{\mathbf{2}}\right)$ & VDVR $\left(\mathrm{m} / \mathbf{s}^{\mathbf{1}, 75}\right)$ \\
\hline Ensaio A & 1,89 & 32,36 \\
Ensaio B & 1,70 & 31,87 \\
\hline
\end{tabular}

Fonte: Autores (2019).

\section{CONCLUSÕES}

Conforme os critérios estabelecidos pela Fundacentro com a análise dos valores aferidos nas avaliações foi possível concluir que os tratores agrícolas com o implemento subsolador em ambas 


\section{$\mathbf{X I X}$ \\ CENEMI}

as condições operacionais apresentaram valores de aceleração e dose de vibração resultante que podem ser prejudiciais aos operadores durante a jornada diária de trabalho. O valor da aceleração resultante da exposição normalizada foi superior a $1,1 \mathrm{~m} / \mathrm{s}^{2}$ e o valor da dose de vibração resultante excedeu o limite de $21 \mathrm{~m} / \mathrm{s}^{1,75}$.

Melhorias relacionadas ao sistema de amortecimento dos tratores agrícolas e nos assentos devem ser consideradas objetivando-se à prevenção de doenças ocupacionais que possam acometer os operadores.

\section{REFERÊNCIAS}

ASSOCIAÇÃO BRASILEIRA DE ERGONOMIA - ABERGO. (2000). Definição Internacional de Ergonomia.

CUNHA, I. DE Â.; GIAMPAOLI, E. Norma de Higiene Ocupacional (NHO 09): Avaliação da Exposição Ocupacional a Vibrações de Corpo Inteiro. São Paulo: Fundacentro, 2013.

FOOD AND AGRICULTURE ORGANIZATION OF THE UNITED NATIONS -.Sustainable Agricultural Mechanization. 2016.

IIDA, I.; BUARQUE, L. Ergonomia: Projeto e Produção. 3. ed. [s.I.] Edgard Blucher, 2016.

INTERNATIONAL ORGANIZATION FOR STANDARDZATION. ISO 8041-1: Human response to vibration - Measuring instrumentation. Geneva, 2005.

RAO, S. Vibrações Mecânicas. São Paulo: [s.n.].

R DEVELOPMENT CORE TEAM. R: A language and environment for statistical computing. Vienna, Austria R Foundation for Statistical Computing, 2018.

SALIBA, T. M. Manual Prático de Avaliação e Controle de Vibração. 4. ed. São Paulo: Ltr Editora Ltda, 2016.

SOUZA, L. H. et al. Níveis de ruído emitidos por uma recolhedora-trilhadora de feijão. Engenharia Agrícola, v. 24, n. 3, p. 745-749, 2004.

VENDRAME, A. C. Perícias Judiciais de Insalubridade e Periculosidade. 4. ed. rev. amp. São Paulo: Ed. do autor, 2017. 355 p.

\section{Agradecimentos}

Ao Conselho Nacional de Desenvolvimento Científico e Tecnológico (CNPq) pelo financiamento da pesquisa (Edital Universal no 01/2016), processo 400500/2016-7. 